\title{
Changes in Safety Attitude and Improvement of Multidisciplinary Teamwork by Implementation of the WHO Surgical Safety Checklist in University Hospital
}

\author{
Takanori Ayabe ${ }^{1,2 *}$, Genji Shinpuku33, Masaki Tomita1, Sayoko Nakamura², Etsuko Yokoyama², \\ Shigeko Shimizu2 ${ }^{2}$, Manabu Okumura², Koichiro Itai ${ }^{3}$, Isao Tsuneyoshi ${ }^{4}$, Hideo Takeshima ${ }^{2,5}$, \\ Kunihide Nakamura ${ }^{2,6}$
}

\begin{abstract}
${ }^{1}$ Division of Thoracic and Breast Surgery, Department of Surgery, Faculty of Medicine, University of Miyazaki, Miyazaki, Japan ${ }^{2}$ Division of Medical Safety Management, Faculty of Medicine, University of Miyazaki, Miyazaki, Japan

${ }^{3}$ Division of Clinical Ethics, Faculty of Medicine, University of Miyazaki, Miyazaki, Japan

${ }^{4}$ Department of Anesthesiology, Faculty of Medicine, University of Miyazaki, Miyazaki, Japan

${ }^{5}$ Department of Neurosurgery, Faculty of Medicine, University of Miyazaki, Miyazaki, Japan

${ }^{6}$ Division of Cardiovascular Surgery, Department of Surgery, Faculty of Medicine, University of Miyazaki, Miyazaki, Japan

Email: ^tayabe@med.miyazaki-u.ac.jp
\end{abstract}

How to cite this paper: Ayabe, T., Shinpuku, G., Tomita, M., Nakamura, S., Yokoyama, E., Shimizu, S., Okumura, M., Itai, K., Tsuneyoshi, I., Takeshima, H. and Nakamura, K. (2017) Changes in Safety Attitude and Improvement of Multidisciplinary Teamwork by Implementation of the WHO Surgical Safety Checklist in University Hospital. Open Journal of Safety Science and Technology, 7, 22-41.

https://doi.org/10.4236/ojsst.2017.71003

Received: January 9, 2017

Accepted: February 13, 2017

Published: February 16, 2017

Copyright $\odot 2017$ by authors and Scientific Research Publishing Inc. This work is licensed under the Creative Commons Attribution International License (CC BY 4.0).

http://creativecommons.org/licenses/by/4.0/

\begin{abstract}
Background: We investigated the changes in consciousness of operating team staffs and the influences on the operating time after the implementation of the surgical safety checklist (SSC) using a questionnaire survey. Materials and Methods: 206 operating team staffs (148 surgeons, 20 anesthesiologists, 38 nurses) replied to the questionnaire survey, and the changes in consciousness were checked before and after the implementation of the SSC. We retrospectively investigated the operating time from medical and anesthesia records at the point 2 months before (pre-implementation (pre-I): $\mathrm{n}=656$ ) and 2 months after (post-implementation (post-I): $\mathrm{n}=650$ ) the implementation. We compared the scheduled operating time, the actual operating time, and the ratio between the two groups. Results: We received replies from 156 operating team staffs, i.e., the recovery rate was $75.7 \%$ (156/206). The operating team staffs were interested in 9 items, which included the self-introduction of members, patient referral, surgical procedure, scheduled operating time, predicted blood loss, important matters in the operation, timing of antibiotics, and important matters in anesthesia, and preparation of required materials and equipment. In the multidisciplinary teamwork, they had increased the consciousness of responsibility and communication. There was no significant difference in the scheduled operating time (post-I: $186.9 \pm 131.9$; pre-I: $184.8 \pm 127.8$ minutes) and the actual operating time (post-I: $170.8 \pm 148.1$
\end{abstract}


minutes; the pre-I: $174.6 \pm 147.3$ minutes). However, regarding the ratio of the actual operating time to the scheduled operating time, there was a significant difference (the post-I: $0.90 \pm 0.43$; the pre-I: $0.95 \pm 0.45$ ). Use of the SSC significantly decreased the actual operating time. Conclusion: The outcomes of the implementation of SSC resulted in changes in the safety consciousness of the operating team staffs such as their increased responsibility and communication. The improved multidisciplinary teamwork might make them realize a smooth operating progression to shorten the actual operating time.

\section{Keywords}

Surgical Safety Checklist, Teamwork, Consciousness, Multidisciplinary

\section{Introduction}

Since the publication of "To Err is Human" [1], worldwide awareness of medical errors has driven the need to control it in the best possible ways. Medical error has been defined as an unintended act (either of omission or commission) or one that does not achieve its intended outcome [2], the failure of a planned action to be completed as intended (an error of execution), the use of a wrong plan to achieve an objective (an error of planning) [3], or a deviation from the process of care that may or may not cause harm to the patient [4]. Patient harm due to a medical error can occur at the individual or system level. Medical error is not included on death certificates or in rankings of the cause of death. Makary M.A. et al. [5] assessed its contribution to mortality. Comparing their estimate to the Centers for Disease Control and Prevention, rankings suggested that medical error is the third most common cause of death in the US [5].

To reduce avoidable morbidity, mortality, and medical errors, safety checklists are designed to improve surgical safety by influencing wider aspects of performance in the operating room, that is, fostering better inter professional teamwork and communication [6]. Breakdowns in multidisciplinary teamwork in the operating room are reported as one of the most common contributory factors towards the occurrence of wrong site surgeries and other surgical adverse events [7] [8] [9] [10] [11].

Recently, based on the guideline of safety surgery by the World Health Organization (WHO) [12], the WHO surgical safety checklist has been implemented on a global scale, which results have been published [13] [14].

In October, 2010, the surgical safety checklist had not been implemented in our University of Miyazaki Hospital. As a hospital working standard of "from the surgical patient's carrying in to the skin incision", the workflow was created by our division of medical patient safety, which had been originally operated. The workflow contains 3 steps, that is, 1) surgical patient's preparation step at arrival from inpatient ward, 2) confirmation steps in front of the operating room entrance, and 3) patient confirmation steps before induction of anesthesia to 
skin incision in the operating room. The surgical patients must be confirmed by documents and personal data assistance. At that time of the timeout section, a simple timeout checklist, which had been performed by an operator, the confirmation items contain the patient's name, procedure, and surgical site (right or left), and the anesthetist and circulating nurse checked the presented information by confirming the informed consent document format. Currently, the level of surgical safety management seemed to be very low.

Belatedly, in Japan, in May, 2012, a WHO surgical safety checklist (Japanese translation version) was introduced at the Seminar of Medical Patient Safety of National, Public, and Private University Hospitals (in Osaka, 2012), which was selected as the fact-finding survey and the mutual check was performed on a national scale.

The implementation of the WHO surgical safety checklist decreased the mortality and the morbidity in surgery and perioperative complications [15] [16] [17] [18] [19]. These checks are designed to minimize the risk of complications and death by reinforcing and standardizing accepted safety procedures (which can be overlooked by a busy team) and by creating a redundancy in the system to allow for human error to be captured [6].

However, there was few studies on the recognition of the medical staffs, such that there is some benefit for patients but questionable as to whether or not there has some benefit for the medical staff. We hypothesized that the implementation of the surgical safety checklist improved the safety attitude of the surgical team staff, that is, a good patient-information grasp by the operating room staffs, sharing of matters, improvement of consciousness for the preparing of equipment, the smooth operating progression, shortening of the operating time and overtime work. We verified these changes in the medical staff's safety attitude, consciousness, recognition, and incidental content before and after the implementation of the surgical safety checklist.

We report an experience during the implementation of the WHO surgical safety checklist in September, 2012, and the outcomes on the changes in safety, attitude and improvement of multidisciplinary teamwork.

\section{Materials and Methods}

\subsection{Approach of the Implementation of the Surgical Safety Checklist and the Timeout Moderation}

The implementation of the surgical safety checklist should be approved in advance by the governing council of the University of Miyazaki Hospital. After we obtained comments and consensus of the implementation at the hospital liaison conference, we informed doctors and nurses of the outcome.

In June, 2012, based on medical evidence from world-wide situations [13] [14], a need for the implementation of the surgical safety checklist was introduced at the monthly-held risk manager conference. At the hospital operating room conference, the implementation method was explained to the members of each of the hospital's medical directors. 
In July, 2012, at the hospital liaison conference, the members that include the hospital director, deputy director, hospital medical director, clinic director, head nurses, deputy minister, deputy director of each clinical department, administrative divisions, launching of the task force on the implementation of the surgical safety checklist was approved. Based on the leadership of the professor in the Department of Anesthesiology, implementation of the surgical safety checklist was decided. After the video-shooting of appearance of timeout and the approval of its step description, and the moderator of time out was decided to hang out by an anesthesiologist.

In August, 2012, the implementation of the surgical safety checklist was approved by the hospital medical safety commission, the step description and a video providing the timeout were featured at the hospital liaison conference.

In September, 2012, the surgical safety checklist was started in the interim, which was finally approved by the governing council of the University of Miyazaki Hospital.

In February, 2013, as a hospital unified rule, the marking method for the surgical site was approved to be added, and the version of the surgical safety checklist for local anesthesia was also approved.

\subsection{Study Protocol}

Table 1 shows our hospital's version of the modified WHO surgical safety checklist. Before a skin incision, all operative team staffs must agree to stop and coordinately run a self-introduction and verify the patient information and sharing of the procedural information by the surgical safety checklist. Table 2 shows our hospital's scenario of time out and moderation procedure (denoted in the left row of Table 2), which represented 9 items of self-assessment (1) self-introduction of members; 2) Patient referral; 3) Surgical procedure; 4) Scheduled operating time; 5) Predicted blood loss; 6) Important matters regarding the operation; 7) Timing of antibiotics; 8) Important matters of anesthesia; 9) Preparation of required materials and equipment) (denoted in the right row of Table 2). The time out is started from a calling by the anesthesiologist. A circulating nurse leaves to record the results of the timeout. The ethics committee in our hospital approved this clinical study.

\subsection{Questionnaire Survey}

Table 3 shows the questionnaire survey for operating team staffs. We surveyed changes in consciousness before and after the implementation of the surgical safety checklist. The survey subjects have to know the changed items in the time out procedure and recognition. Our operating team staffs (surgeons, anesthesiologist, and nurses) replied to three questions in the questionnaire survey by a self-assessment at 2 months after implementation of the surgical safety checklist.

Question 1 (Q. 1) is to evaluate 9 items, which are based on the scenario and each part of the moderation by the anesthesiologist in Table 2 (in the left and 
Table 1. Surgical safety checklist in University of Miyazaki Hospital.

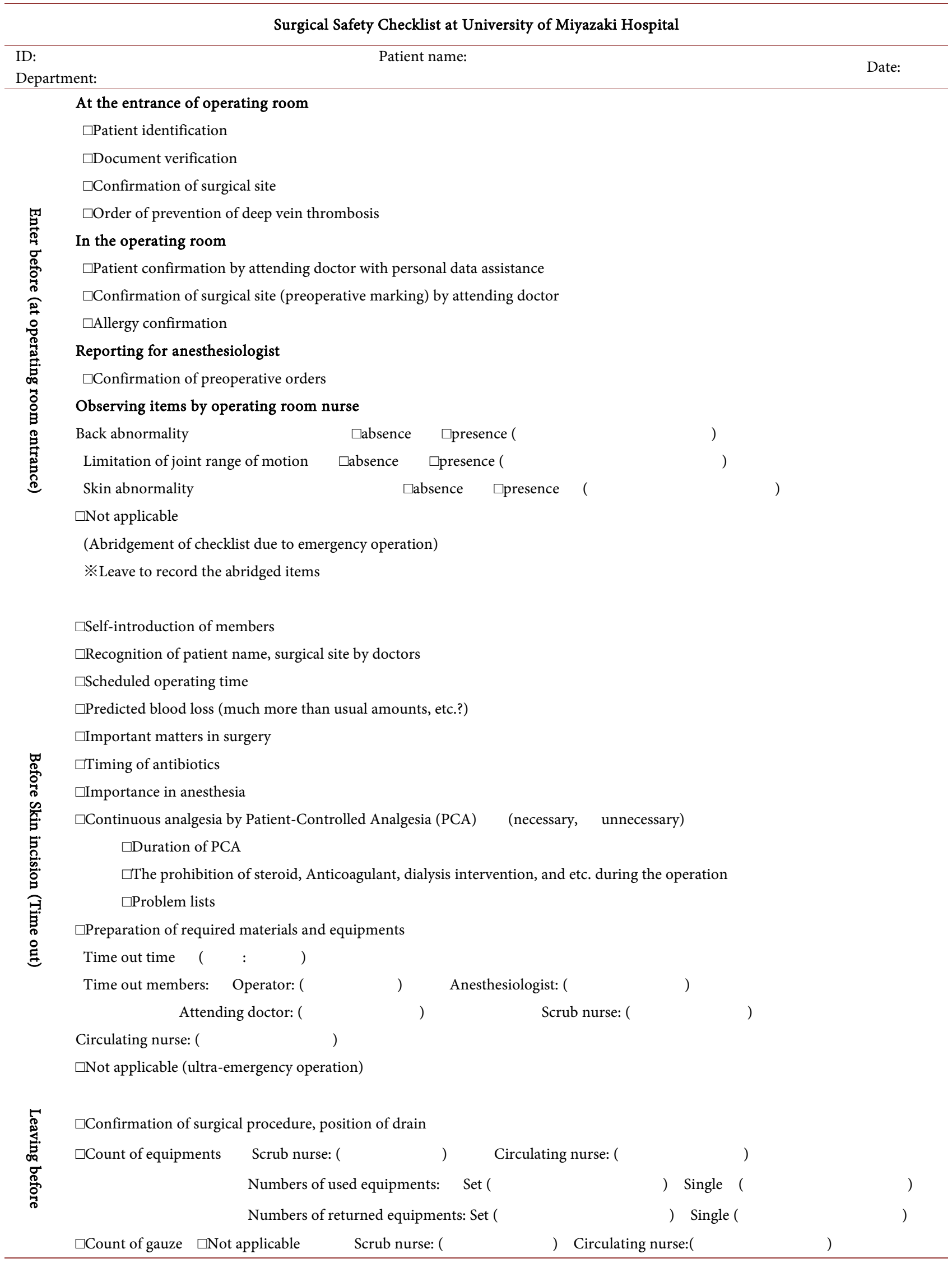




\section{Continued}

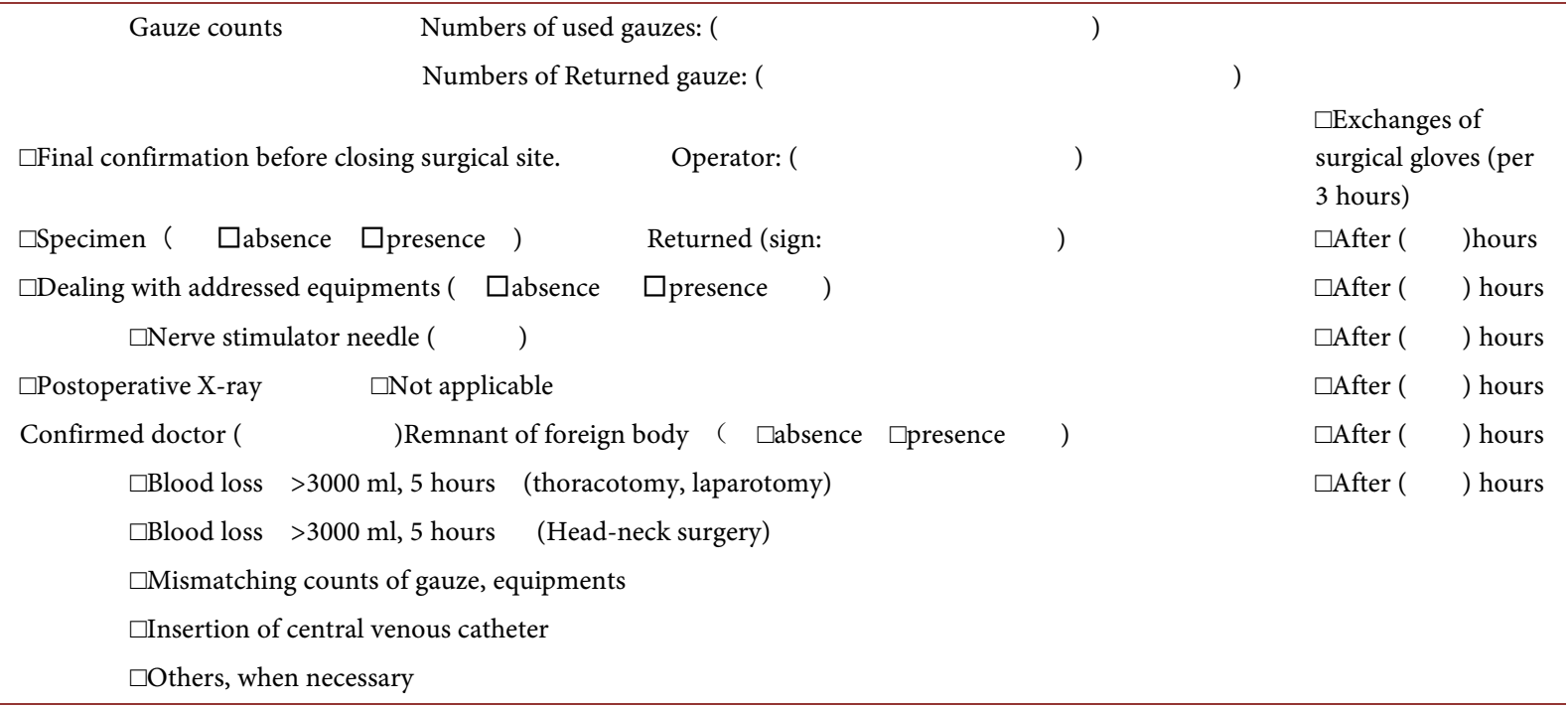

*Starting from calling by anesthesiologist. Department of operation, University of Miyazaki Hospital.

Table 2. Procedure of time out (moderated by anestheliogist) and 9 items of self-assessment.

Procedure of time out (moderated by anesthesiologist)

1 Anesthesiologist: I will start time out from now.

Anesthesiologist: I am an anesthetist, "name". Please introduce your name, in the order of operator, attending doctor, the first assistant, the second assistant, scrub nurse, circulating nurse, and students.

2 Team members: I'm an operator, "NAME". I'm an attending doctor, "NAME". I'm a first assistant. I'm a second assistant, "NAME". I'm a scrub nurse, "NAME". I'm a circulating nurse, "NAME". I'm a student, "NAME".

Anesthesiologist: Let me know about the patient's name, procedure.

3 Operator: The Patient is "NAME". The incision is right or left, ○०००.

Anesthesiologist: Let me know about the scheduled operating time and the anticipated blood loss.

Operator: Operating time is $(\mathrm{X})$ hours, blood loss is about $(\mathrm{Y}) \mathrm{ml}$.

Anesthesiologist: What are the critical or non-routine steps?

Operator: I might perform ...

Anesthesiologist: I've gave the first antibiotic prophylaxis. Has

6 antibiotic prophylaxis been given in the next?

Operator: nothing. (or every 6 hours later)

7 Anesthesiologist: There are some patient-specific concerns.

Anesthesiologist: Has sterility been confirmed? Are there equipment matters or any concerns?

Scrub and circulating nurses: Yes, sterility has been confirmed. There are equipment issues.

Anesthesiologist: I'm looking forward to working with you.

All team members: I'm looking forward to working with you.
9 items of self-assessment

1. Self-introduction of

members

2. Patient referral

3. Surgical procedure

4

Scheduled operating time

5. Predicted blood loss

6. Important matters in operation

7. Timing of antibiotics

8. Important matters in anesthesia

Preparation of re-

9. quired materials and equipment 
Table 3. The questionnaire survey.

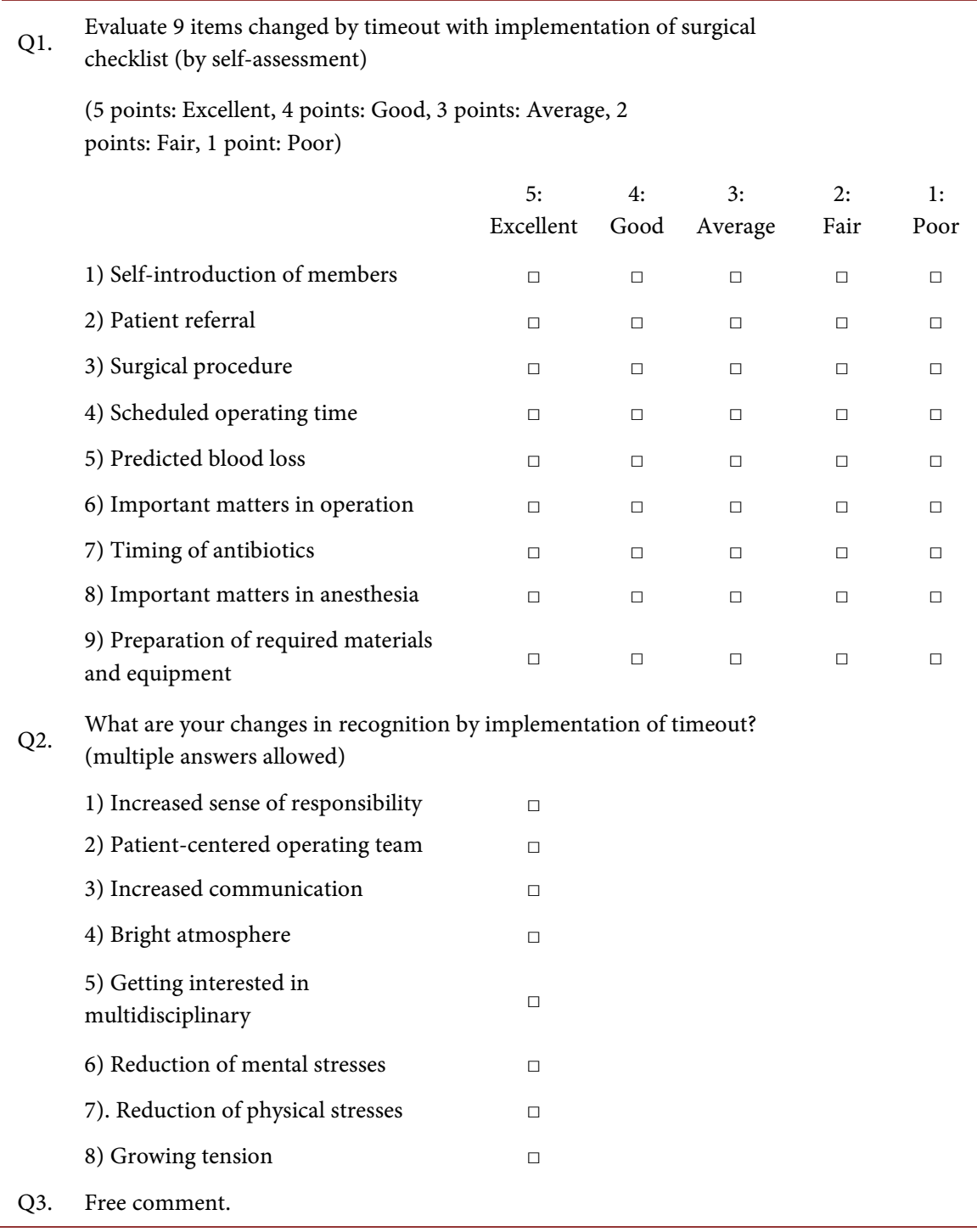

right rows of Table 2). The 9 items on the timeout procedure are 1) self-introduction of members, 2) patient referral, 3) surgical procedure, 4) scheduled operating time, 5) predicted blood loss, 6) important matters about the operation, 7) timing of antibiotics, 8) important matters about anesthesia, and 9) preparation of required materials and equipment. These 9 items on the timeout procedure were checked by a multidisciplinary self-assessment. Before and after implementation of the surgical safety checklist, the items were replied by a 5 -stage evaluation (5 points: excellent, 4 points: good, 3 points: average, 2 points: fair, 1 point: poor). Question 2 (Q. 2) is to answer what recognition of the 8 items to be changed is due to the implementation of the surgical safety checklist. Before and after the implementation, the recognition configured 8 items, which are 1) increased sense of responsibility, 2) patient-centered operative team, 3) increased communication, 4) bright atmosphere, 5) getting interested in multi-disciplinary, 6) reduction of mental stress, 7) reduction of physical stress, and 8) grow- 
ing tension. Multiple answers can be allowed. Question 3 (Q. 3) is to add free comments.

\subsection{Retrospective Analysis of Medical and Anesthesia Record}

Retrospectively, we randomly evaluated hundreds of patients who underwent surgery in our hospital. We investigated gender, age, body height, body weight, scheduled operating time, anesthesia time, actual operating time, ratio of actual operating time to the scheduled operating time, case numbers that extended over the scheduled time, and time in the operating room over the scheduled time, based on the medical and anesthesia records at 2 months before and after the implementation of the surgical safety checklist. Regarding these two points (preimplementation and post-implementation), we compared the above parameters between the two groups.

\subsection{Statistical Analysis}

Statistical differences in the parameters between the two groups were calculated using the t-test or the Mann-Whitney test. A $p$ value less than 0.05 was considered to be statistically significant.

\section{Results}

\subsection{Outcomes of Multidisciplinary Self-Assessment of 9 Items on Timeout by Implementation of Surgical Safety Checklist (Question 1)}

We requested a bearer questionnaire for 206 operating team staffs (148 surgeons, 20 anesthesiologists, 38 nurses). As a result, we received replies from 156 operating staffs (119 surgeons, 13 anesthesiologists, 24 nurses) and the recovery rate was $75.7 \%(156 / 206)$.

Figure 1 to Figure 4 show the results of 9 items evaluated by the self-assessment before and after the implementation of the surgical safety checklist. The highest items that displayed more than 4.0 points (more than "4: good") by the self-assessment were denoted in the darker colors of the 9 items.

Figure 1 shows that surgeons $(\mathrm{n}=119)$ evaluated all 9 items as a display of more than a 3.84-point, that is nearer to the 4.0-point(3-point is "average"; 4 -point is "good") and evaluated 6 items of which were more than the 4.0-point (more than "good"), that were the "self-introduction of members" (4.04-point), "scheduled operating time" (4.08-point), "predicted blood loss" (4.03-point), "important matters regarding the operation" (4.14-point), "timing of antibiotics" (4.08-point), and "important matters in anesthesia" (4.15-point), especially, the highest points of which were the items of "important matters regarding the operation" (4.14-point) and "important matters about anesthesia" (4.15-point), and the lowest points of which were the items of "patient referral" (3.84-point) and the "surgical procedure" (3.84-point).The total points were 36.12-and the averaged point was 4.013(36.12/9). The outcomes in surgeons represented "almost good" because that 9 items showed the around 4-point. 
9. Preparation of required materials and equipment

8. Important matters in anesthesia

7. Timing of antibiotics

6. Important matters in operation

5. Predicted blood loss

4. Scheduled operating time

3. Surgical procedure

2. Patient referral

1. Self-introduction of members

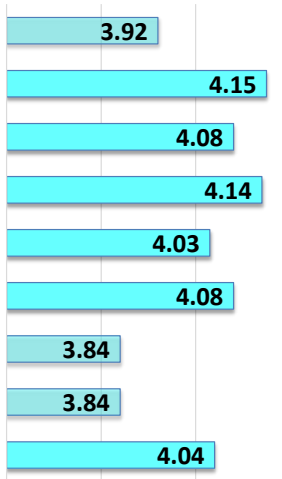

3.6

3.8

4

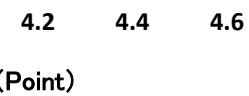

4.8

Figure 1. Q. 1. Self-assessment of 9 items on time out by implementation of surgical safety checklist (Outcomes for surgeons).

9. Preparation of required materials and equipment

8. Important matters in anesthesia

7. Timing of antibiotics

6. Important matters in operation

5. Predicted blood loss

4. Scheduled operating time

3. Surgical procedure
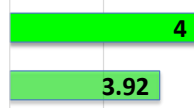

2. Patient referral

1. Self-introduction of members
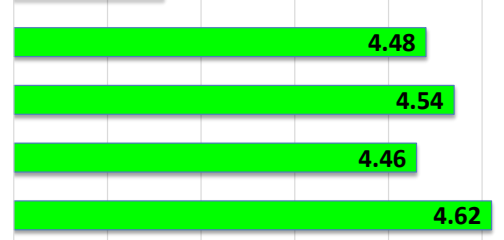

4.62
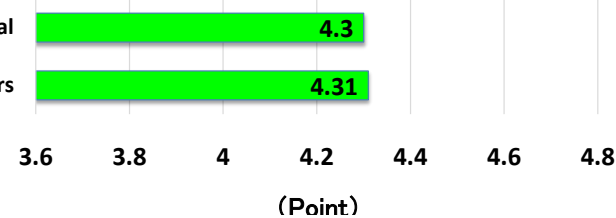

Figure 2. Q. 1. Self-assessment of 9 items on time out by implementation of surgical safety checklist (Outcomes for anesthesiologists).

9. Preparation of required materials and equipment

8. Important matters in anesthesia

7. Timing of antibiotics

6. Important matters in operation

5. Predicted blood loss
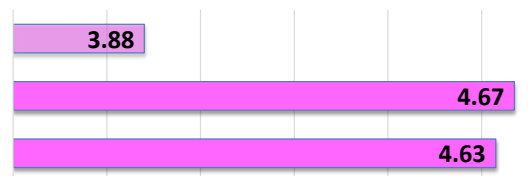

4.71

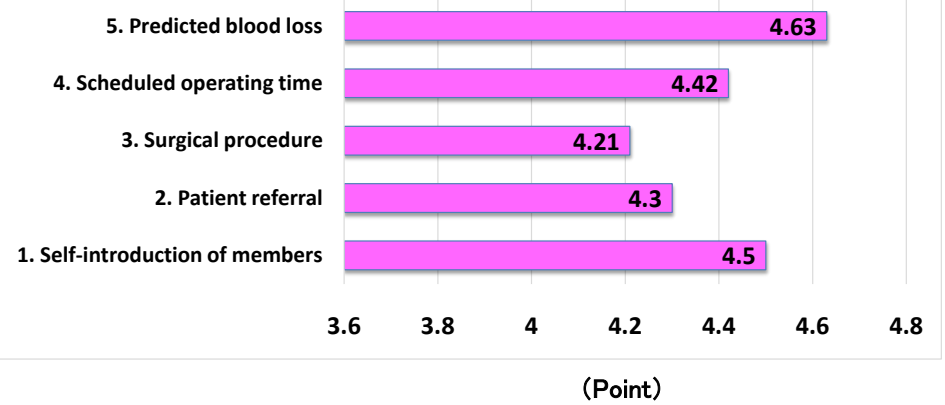

Figure 3. Q. 1. Self-assessment of 9 items on time out by implementation of surgical safety checklist (Outcomes for nurses). 
9. Preparation of required materials and equipment

8. Important matters in anesthesia

7. Timing of antibiotics

6. Important matters in operation

5. Predicted blood loss

4. Scheduled operating time

3. Surgical procedure

2. Patient referral

1. Self-introduction of members

3.6

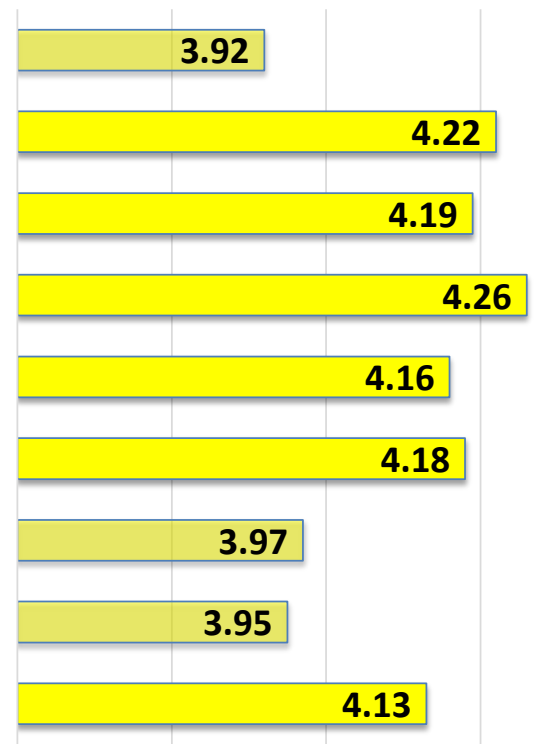

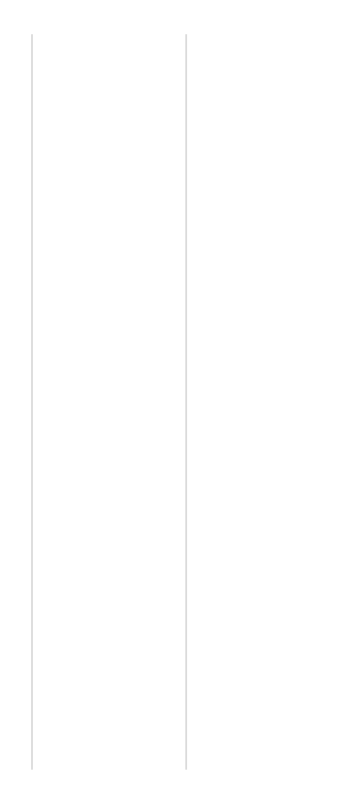

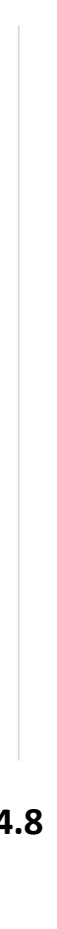

Figure 4. Q. 1. Self-assessment of 9 items on time out by implementation of surgical safety checklist (Outcomes for multidisciplinary).

Figure 2 showed that anesthesiologists $(n=13)$ evaluated all 9 items as a display of more than a 3.92-point, which is much nearer to the 4.0-point (3-point is "average"; 4-point is "good") and evaluated 8 items of which showed more than 4.0-point (more than "good"), which were the "self-introduction of members" (4.31-point), "patient referral" (4.3-point), "surgical procedure” (4.62-point), "scheduled operating time" (4.62-point), "predicted blood loss" (4.46-point), "important matters regarding the operation" (4.54-point), "timing of antibiotics" (4.48-point), and "preparation of required materials and equipment" (4-point). The total points were 39.25- and the averaged point was 4.361 (39.25-point/9). The outcomes by the anesthesiologists seemed to focus on almost all items and represented a situation of more than "good", even though the lowest points, which was the item of "important matters in anesthesia" (3.92-point), is very close to the 4.0-point ("good").

The above points obtained by the anesthesiologists (total point: 39.25) were relatively higher than those of the surgeons (total point: 36.12) (Figure 1). The anesthesiologists suggested that the use of the surgical safety checklist changed the safety attitude in the operating room compared to those of the surgeons.

Figure 3 showed that nurses $(n=24)$ evaluated all 9 items as a display of more than 3.8 points(3-point is "average"; 4-point is "good") and had high interests in 7 items of which showed more than the 4.0-point(more than "good"), which were the "self-introduction of members" (4.5-point), "patient referral" (4.3-point), "surgical procedure" (4.21-point), "scheduled operating time" (4.42-point), "predicted blood loss" (4.63-point), "important matters regarding the operation" (4.71- 
point), "timing of antibiotics" (4.63-point), and "important matters about anesthesia" (4.67-point). The total points were 39.95 -points, and the averaged point was 4.439 (39.95-point/9). The outcomes for the nurses had high interests in almost all items, however, the lowest points was the item of "preparation of required materials and equipment" (3.88-point).

The above points obtained by nurses (total point: 39.95$)$ were relatively higher than those of the surgeons (total point: 36.12) (Figure 1) and were similar to those of the anesthesiologists (total point: 39.25) (Figure 2). The anesthesiologists and nurses suggested that the use of the surgical safety checklist changed the safety attitude in the operating room compared to those of the surgeons.

Figure 4 showed that multidisciplinary $(n=156)$ evaluated all 9 items of a display of more than 3.9 points (3-point is "average"; 4-point is "good"), and 6 items of which displayed more than 4.0 points (more than "good"), that were the "self-introduction of members" (4.13-point), "scheduled operating time" (4.18point), "predicted blood loss" (4.16-point), "important matters regarding the operation" (4.26-point), "timing of antibiotics" (4.19-point), and "important matters in anesthesia" (4.22-point). As the ratio of surgeons was $57.8 \%(119 / 206)$ of the replied staffs, the results of the multidisciplinary appeared to be similar and depended on those of the surgeons.

The above points obtained by the multidisciplinary (total point: 36.98 ) were similar to those of the surgeons (total point: 36.12) (Figure 1). The anesthesiologists (total point: 39.25 ) and nurses (total point: 39.95 ) suggested that the use of the surgical safety checklist changed the safety attitude in the operating room compared to those of the surgeons.

Summarizing the above results according to each occupation, the items focused on by the surgeons, anesthesiologists, and nurses were different, the outcomes of which seemed to be interesting, however, those differences appeared to represent each of the professional's characteristics and their responsibilities.

\subsection{What Are Your Changes in Recognition by the Implementa- tion of the Surgical Safety Checklist? (Multiple Answers Al- lowed) (Question 2)}

Figure 5 to Figure 8 show the replied ratio (percentage) and results of the selfassessment of 8 items on the changes in recognition by the implementation of the surgical safety checklist. The highest items displayed accumulated by the multiple answers allowed were denoted by the darker colors in the 8 items.

Figure 5 showed that the surgeons $(n=119)$ had a higher interest in the item of "increased sense of responsibility" (40.3\%) and the "increased communication" (37.8\%) than the other items. There was no reply to the "reduction of physical stresses" $(0 \%)$. After the implementation of the surgical safety checklist, the outcomes by surgeons had more focused on the "increased responsibility" and the "increased communication".

Figure 6 showed that the anesthesiologists $(\mathrm{n}=13)$ had a much higher interest in the "increased communication" (53.8\%). There was no reply to the "increased sense of responsibility" (0\%) and the "reduction of physical stresses" 
What are your changes in recognition by implementation of time out? (multiple answers allowed)

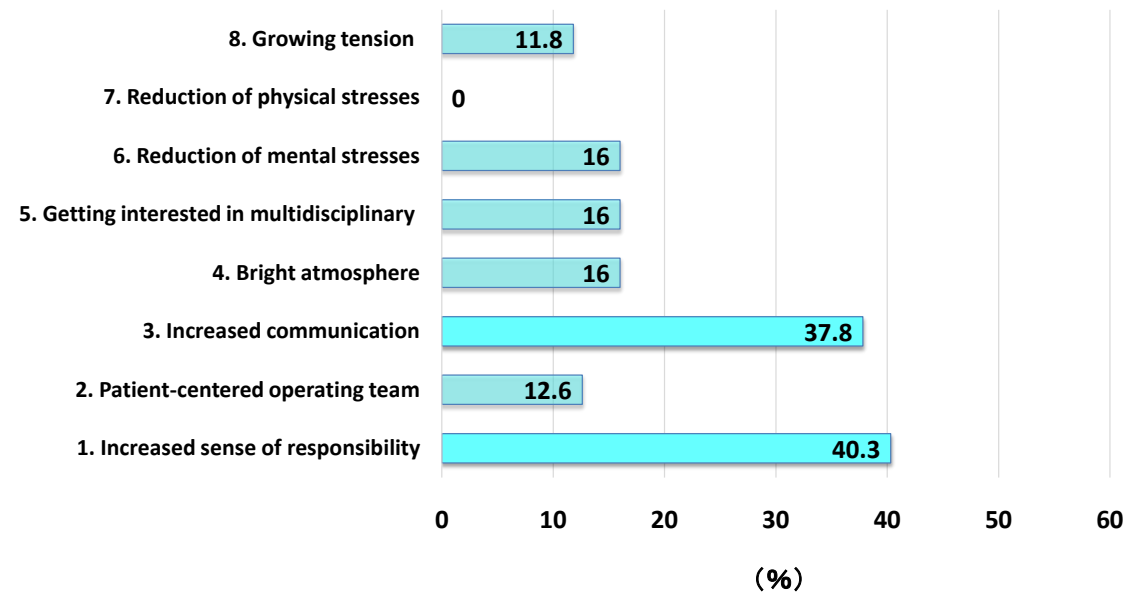

Figure 5. Q. 2. Self-assessment of 9 items on time out by implementation of surgical safety checklist (Outcome for surgeons).

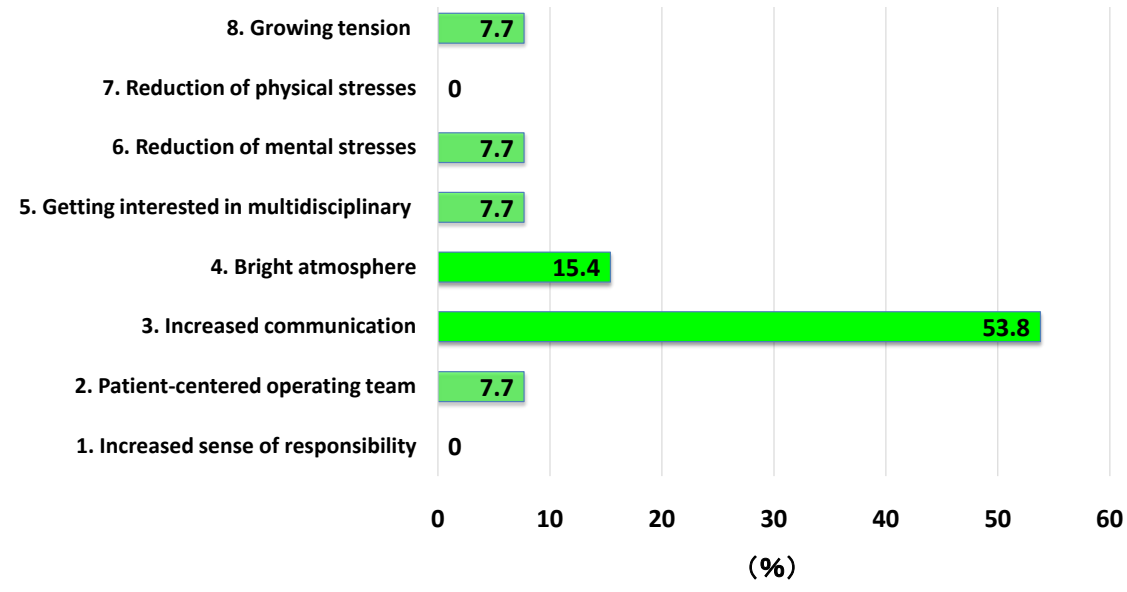

Figure 6. Q. 2. Self-assessment of 9 items on time out by implementation of surgical safety checklist (Outcomes for anesthesiologists).

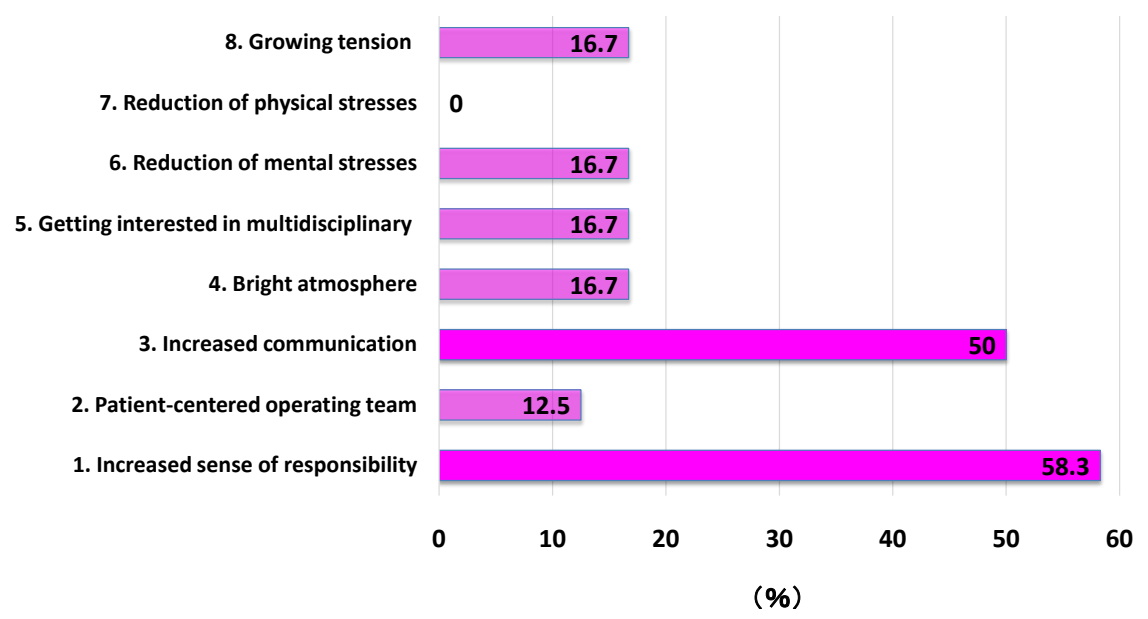

Figure 7. Q. 2. Self-assessment of 9 items on time out by implementation of surgical safety checklist (Outcomes for nurses). 


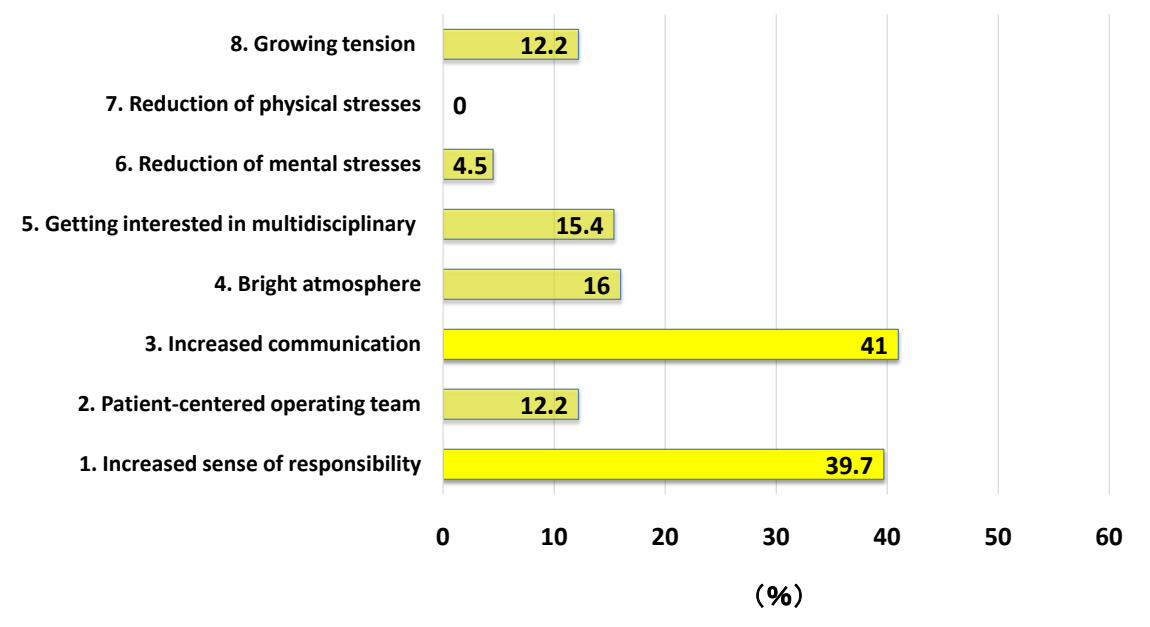

Figure 8. Q. 2. Self-assessment of 9 items on time out by implementation of surgical safety checklist (Outcomes for multidisciplinary).

(0\%). The outcomes by the anesthesiologists had more focused on the "increased communication".

Figure 7 showed that nurses $(n=24)$ had a high interest in the "increased sense of responsibility" (58.3\%) and "increased communication" (50\%). There was no reply to the "reduction of physical stresses" $(0 \%)$. The outcomes by the nurses resulted in the high interest in the "increased responsibility" and "increased communication".

Figure 8 showed that multidisciplinary $(\mathrm{n}=156)$ of the operating team staffs had a high interest in the "increased sense of responsibility" (39.7\%) and "increased communication" (41\%). There was no reply to the "reduction of physical stresses" (0\%). The outcomes in multidisciplinary had significantly focused on the "increased responsibility" and the "increased communication".

Summarizing these replied results according to each occupation, the items focused by surgeons, anesthesiologists, and nurses were different, however, one common outcome seemed to be focused on the 'increased communication' and the use of a surgical safety checklist appeared to contribute to the operative staffs to construct a good communication, teamwork, and collaboration in the operating room.

\subsection{Free Comments (Question 3)}

There were some free comments, such as building of a patient-centered team, good atmosphere in communication between the operating team staffs, increased communication by the operating team, good work atmosphere, increased payment of attention to multidisciplinary task and work, and reduction of mental stresses and physical stresses.

\subsection{Retrospective Analysis of Medical and Anesthesia Records}

Table 4 displays the results of the investigation based on the medical and anesthesia records. At the point of 2 months before and after the implementation of 
Table 4. Outcomes before and after the implementation of surgical safety checklist.

\begin{tabular}{ccc}
\hline Outcomes & Pre-implementation & Post-implementation \\
\hline Case numbers & 656 & 650 \\
Gender (Male:Female) & $340: 316$ & $356: 294$ \\
Age & $45.2 \pm 26.2$ & $51.7 \pm 24.8$ \\
Body height (cm) & $151.6 \pm 21.5$ & $152.1 \pm 22.8$ \\
Body weight (kg) & $52.6 \pm 18.0$ & $54.1 \pm 18.1$ \\
$\begin{array}{c}\text { Scheduled operating time (min) } \\
\text { Anesthesia time (min) } \\
\text { Actual operating time (min) }\end{array}$ & $184.8 \pm 127.8$ & $186.9 \pm 131.9$ \\
$\begin{array}{c}\text { Ratio of actual operating time to the scheduled } \\
\text { operating time }\end{array}$ & $236.5 \pm 168.0$ & $229.9 \pm 170.5$ \\
Case numbers that extended over the \\
scheduled time (min)
\end{tabular}

the surgical safety checklist, we randomly extracted656 medical and anesthesia records (as a pre-implementation group: pre-I group) and 650 ones (as a postimplementation group: post-I group). We compared these two groups.

There was a significance in ages between the two groups (pre-I group: $45.2 \pm$ 26.2 , and post-I group: $51.7 \pm 24.8$ ). Between the two groups, there was no significance regarding body height, body weight, anesthesia time, scheduled operating time, and actual operating time. For the scheduled operating time, $186.9 \pm$ 131.9 minutes in the post-I group was longer compared to that in the pre-I group (184.8 \pm 127.8 minutes).

For the anesthesia time, $229.9 \pm 170.5$ minutes in the post-I group was shorter compared to that in the pre-I group (236.5 \pm 168.0 minutes), but there was no significance. For the actual operating time, $170.8 \pm 148.1$ minutes in the post-I group was shorter compared to that for the pre-I group (174.6 \pm 147.3 minutes), but there was no significance between the two groups.

However, for the ratio of the actual operating time to the scheduled operating time, $0.90 \pm 0.43$ in the post-I group was shorter compared than that for the pre-I group $(0.95 \pm 0.45)$, and there was a significance. The use of the surgical safety checklist significantly decreased the actual operating time after the implementation.

For the case numbers that extended over the scheduled time, that in the post-I group ( $\mathrm{n}=148,148 / 650,22.8 \%)$ resulted in less compared to that in the pre-I group ( $\mathrm{n}=165,165 / 656,25.2 \%)$ (there was no significance). The use of the surgical safety checklist might display a tendency of fewer case numbers that extended over the scheduled time after the implementation.

For the staying time in the operating room over the scheduled time, that in the post-I group (118.7 \pm 7.5 minutes) was shorter compared to that in the pre-I group (121.6 \pm 7.6$)$ (there was no significance). The use of the surgical safety 
checklist might display a tendency of a shorter staying time in the operating room over the scheduled time after the implementation.

\section{Discussion}

Every year, an estimated 234 million surgeries are performed globally. Of these, an estimated seven million complications and one million deaths occur [20], half of which are thought to be preventable [21] [22] [23].

Preventable errors are often not related to technical skill failure, training, or knowledge but represent cognitive, system or teamwork failure [3] [24] [25] [26] [27]. Nontechnical skills, such as communication, cooperation, coordination, and leadership, are critical components of teamwork, but limited interpersonal skills often underlie adverse events and errors [28] [29] [30]. In a review of litigated surgical outcomes, communication failures accounted for $87 \%$ of the system failures that led to an indemnity payment [31]. The communication failure occurred primarily between caregivers, rather than between the caregiver and patient.

One strategy that is being promoted to help reduce mortality and complications that arise during surgery is the use of surgical safety checklists [14]. The World Health Organization (WHO) Surgical Safety Checklist was developed through its Safe Surgery Saves Lives Campaign [12]. Central to this campaign is the WHO Surgical Safety Checklist, a 19-item checklist that promotes safety by helping clinicians adhere to an evidence-based best practice [15]. In its pilot program, the checklist was found to decrease mortality and complications by 48 and 37\%, respectively, in eight hospitals around the world [16].

The exact mechanism by which surgical checklists improve patient outcomes is not well understood, but it is thought that multiple factors play a role in this. The key factors include improved team communication [32] [33] [34], teamwork [16] [33] [35], compliance with prophylactic antibiotic administration [14] [36] [37] and monitoring prior to administration of an aesthesia [38].

Checklists or protocols are a common tool for preventing human errors in complex and high intensity areas of work [21]. The use of a checklist is associated with changes in systems and changes in the culture of operating theater teams [25] [39]. These cultural changes increase communication and teamwork within the surgical team by delegating the responsibility for patient safety to the whole team away from a purely hierarchical system and enhancing the work satisfaction of the health care professionals [40]. In addition, the use of a checklist helps to determine each person's function during the surgical procedure [41] [42].

Several studies have shown that team communication and teamwork are critical factors for patient safety and quality and could prevent many deaths and major complications in surgery [25] [43] [44].

By promoting direct verbal communication and interaction, the checklist aims to open the line of communication between the operating room team members, to ensure a common understanding or "shared mental model" of the pa- 
tient, procedure, and risk, and to empower individuals to voice safety concerns who may not otherwise feel able to do so, thus increasing the probability of surgical error being captured or mitigated before it is too late [6].

The present study showed that the implementation of the surgical safety checklist changed the operating team staffs to increase their consciousness for multidisciplinary, and to increase their professional responsibility and communication. After the implementation, as a result, the ratio of the actual operating time to the scheduled operating time has become significantly lower. This was the reason why the operating team staff began to concentrate their consciousness as a novel medical resource. By the implementation, sufficient understanding of the patient information should provide the operating team staffs with a smooth surgery progression. Their increased responsibility and communication in multidisciplinary have changed their safety attitude, their consciousness for the scheduled operating time that might result in a smooth surgery progression. As a result, such a good affect and situation might shorten the actual operating time.

The surgical safety checklist is a good communication tool for the operating team staff in multidisciplinary, which is also a good briefing and debriefing tool that becomes a surgical patient information sharing system. This includes the scheduled operating time, estimated amount of blood loss, matters in surgery, timing of antibiotics, matters in anesthesia, and preparation of materials and equipment, information that needs to be shared with the operating team staff in multidisciplinary. The implementation of the surgical safety checklist should naturally be in the everyday preoperative briefing with sharing of the operating matters, the preparing of equipment, and the smooth operating progression, which common safety culture improves the surgical teamwork in multidisciplinary.

The limitation of the present study was considered that there were no assessment of complications and mortality, exclusion of the emergent surgery, the evaluation of the changes in consciousness as judged by the self-assessment, and it is a short-term study without a long-term follow-up.

\section{Conclusions}

By implementation of the surgical safety checklist, the consciousness for the operating team staffs and the responsibility and communication increased, which validated the reduction in the actual operating time compared to the scheduled operating time. However, there was no significant change in the operating team staff's working overtime.

The surgical safety checklist is a good communication tool for the operating teamwork and has become a good surgical patient information sharing system.

\section{References}

[1] Kohn, L.T., Corrigan, J., Donaldson, M.S., Eds. (2000) To Err Is Human: Building a Safer Health System. Committee on Quality of Health Care in America, Institute of Medicine, National Academy Press, Washington, DC.

[2] Leape, L.L. (1994) Error in Medicine. JAMA, 21, 1851-1857. 
https://doi.org/10.1001/jama.1994.03520230061039

[3] Reason, J. (1990) Human Error. Cambridge University Press, Cambridge.

[4] Reason, J.T. (2001) Understanding Adverse Events: The Human Factor. In: Vincent C, Ed., Clinical Risk Management. Enhancing Patient Safety, BMJ Books, London, 9-30.

[5] Makary, M.A. and Daniel, M. (2016) Medical Error-The Third Leading Cause of Death in the US. BMJ, 353, i2139. https://doi.org/10.1136/bmj.i2139

[6] Russ, S., Rout, S., Sevdalis, N., Moorthy, K., Darzi, A. and Vincent, C. (2013) Do Safety Checklists Improve Teamwork and Communication in the Operating Room? A Systematic Review. Annals of Surgery, 258, 856-871. https://doi.org/10.1097/SLA.0000000000000206

[7] Neily, J., Mills, P.D., Eldridge, N., Dunn, E.J., Samples, C., Turner, J.R., Revere, A., DePalma, R.G. and Bagian, J.P. (2009) Incorrect Surgical Procedures within and Outside of the Operating Room. Archives of Surgery, 144, 1028-1034. https://doi.org/10.1001/archsurg.2009.126

[8] Greenberg, C.C., Regenbogen, S.E., Studdert, D.M., Lipsitz, S.R., Rogers, S.O., Zinner, M.J. and Gawande, A.A. (2007) Patterns of Communication Breakdowns Resulting in Injury to Surgical Patients. Journal of the American College of Surgeons, 204, 533-540. https://doi.org/10.1016/j.jamcollsurg.2007.01.010

[9] Rogers Jr., S.O., Gawande, A.A., Kwaan, M., Puopolo, A.L., Yoon, C., Brennan, T.A. and Studdert, D.M. (2006) Analysis of Surgical Errors in Closed Malpractice Claims at 4 Liability Insurers. Surgery, 140, 25-33. https://doi.org/10.1016/j.surg.2006.01.008

[10] Williams, R.G., Silverman, R., Schwind, C., Fortune, J.B., Sutyak, J., Horvath, K.D., Van Eaton, E.G., Azzie, G., Potts $3^{\text {rd }}$, J.R., Boehler, M. and Dunnington, G.L. (2007) Surgeon Information Transfer and Communication: Factors Affecting Quality and Efficiency of Inpatient Care. Annals of Surgery, 245, 159-169. https://doi.org/10.1097/01.sla.0000242709.28760.56

[11] Hull, L., Arora, S., Aggarwal, R., Darzi, A., Vincent, C. and Sevdalis, N. (2012) The Impact of Nontechnical Skills on Technical Performance in Surgery: A Systematic Review. Journal of the American College of Surgeons, 214, 214-230. https://doi.org/10.1016/j.jamcollsurg.2011.10.016

[12] WHO (2009) Guidelines for Safe Surgery 2009: Safe Surgery Saves Lives. WHO Guidelines Approved by the Guidelines Review Committee, World Health Organization, Geneva.

[13] Haynes, A.B., Weiser, T.G., Berry, W.R., Lipsitz, S.R., Breizat, A.H., Dellinger, E.P., Herbosa, T., Joseph, S., Kibatala, P.L., Lapitan, M.C., Merry, A.F., Moorthy, K., Reznick, R.K., Taylor, B., Gawande, A.A. and Safe Surgery Saves Lives Study Group (2009) A Surgical Safety Checklist to Reduce Morbidity and Mortality in a Global Population. The New England Journal of Medicine, 360, 491-499. https://doi.org/10.1056/NEJMsa0810119

[14] De Vries, E.N., Prins, H.A., Crolla, R.M., den Outer, A.J., van Andel, G., van Helden, S.H., Schlack, W.S., van Putten, M.A., Gouma, D.J., Dijkgraaf, M.G., Smorenburg, S.M. and Boermeester, M.A. and Surpass Collaborative Group (2010) Effect of a Comprehensive Surgical Safety System on Patient Outcomes. The New England Journal of Medicine, 363, 1928-1937. https://doi.org/10.1056/NEJMsa0911535

[15] Weiser, T.G., Haynes, A.B., Dziekan, G., Berry, W.R., Lipsitz, S.R. and Gawande, A.A. and Safe Surgery Saves Lives Investigators and Study Group (2010) Effect of a 19-Item Surgical Safety Checklist during Urgent Operations in a Global Patient Population. Annals of Surgery, 251, 976-980. 
https://doi.org/10.1097/SLA.0b013e3181d970e3

[16] Haynes, A.B., Weiser, T.G., Berry, W.R., Lipsitz, S.R., Breizat, A.H., Dellinger, E.P., Dziekan, G., Herbosa, T., Kibatala, P.L., Lapitan, M.C., Merry, A.F., Reznick, R.K., Taylor, B., Vats, A. and Gawande, A.A. (2011) Changes in Safety Attitude and Relationship to Decreased Postoperative Morbidity and Mortality Following Implementation of a Checklist-Based Surgical Safety Intervention. BMJ Quality and Safety, 20, 102-107. https://doi.org/10.1136/bmjqs.2009.040022

[17] Bliss, L.A., Ross-Richardson, C.B., Sanzari, L.J., Shapiro, D.S., Lukianoff, A.E., Bernstein, B.A. and Ellner, S.J. (2012) Thirty-Day Outcomes Support Implementation of a Surgical Safety Checklist. Journal of the American College of Surgeons, 215, 766-776. https://doi.org/10.1016/j.jamcollsurg.2012.07.015

[18] Bergs, J., Hellings, J., Cleemput, I., Zurel, Ö., De Troyer, V., Van Hiel, M., Demeere, J.L., Claeys, D. and Vandijck, D. (2014) Systematic Review and Meta-Analysis of the Effect of the World Health Organization Surgical Safety Checklist on Postoperative Complications. British Journal of Surgery, 101, 150-158. https://doi.org/10.1002/bjs.9381

[19] Chaudhary, N., Varma, V., Kapoor, S., Mehta, N., Kumaran, V. and Nundy, S. (2015) Implementation of a Surgical Safety Checklist and Postoperative Outcomes: A Prospective Randomized Controlled Study. Journal of Gastrointestinal Surgery, 19, 935-942. https://doi.org/10.1007/s11605-015-2772-9

[20] Weiser, T.G., Regenbogen, S.E., Thompson, K.D., Haynes, A.B., Lipsitz, S.R., Berry, W.R. and Gawande, A.A. (2008) An Estimation of the Global Volume of Surgery: A Modelling Strategy Based on Available Data. The Lancet, 12, 139-144. https://doi.org/10.1016/S0140-6736(08)60878-8

[21] Kable, A.K., Gibberd, R.W. and Spigelman, A.D. (2002) Adverse Events in Surgical Patients in Australia. International Journal for Quality in Health Care, 14, 269-276. https://doi.org/10.1093/intqhc/14.4.269

[22] Vincent, C., Neale, G. and Woloshynowych, M. (2001) Adverse Events in British Hospitals: Preliminary Retrospective Record Review. BMJ, 322, 517-519. https://doi.org/10.1136/bmj.322.7285.517

[23] Thomas, E.J., Studdert, D.M., Burstin, H.R., Orav, E.J., Zeena, T., Williams, E.J., Howard, K.M., Weiler, P.C. and Brennan, T.A. (2000) Incidence and Types of Adverse Events and Negligent Care in Utah and Colorado. Medical Care, 38, 261-271. https://doi.org/10.1097/00005650-200003000-00003

[24] Reason, J. (2008) The Human Contribution: Unsafe Acts, Accidents and Heroic Recoveries. Ashgate Publishing, Burlington, VT.

[25] Mazzocco, K., Petitti, D.B., Fong, K.T., Bonacum, D., Brookey, J., Graham, S., Lasky, R.E., Sexton, J.B. and Thomas, E.J. (2009) Surgical Team Behaviors and Patient Outcomes. The American Journal of Surgery, 197, 678-685.

https://doi.org/10.1016/j.amjsurg.2008.03.002

[26] Carthey, J., de Leval, M.R. and Reason, J.T. (2001) The Human Factor in Cardiac Surgery: Errors and near Misses in a High Technology Medical Domain. The Annals of Thoracic Surgery, 72, 300-305. https://doi.org/10.1016/S0003-4975(00)02592-3

[27] Shouhed, D., Gewertz, B., Wiegmann, D. and Catchpole, K. (2012) Integrating Human Factors Research and Surgery: A Review. Archives of Surgery, 147, 1141-1146. https://doi.org/10.1001/jamasurg.2013.596

[28] Mishra, A., Catchpole, K., Dale, T. and McCulloch, P. (2008) The Influence of Non-Technical Performance on Technical Outcome in Laparoscopic Cholecystectomy. Surgical Endoscopy, 22, 68-73. https://doi.org/10.1007/s00464-007-9346-1 
[29] Catchpole, K.R., Giddings, A.E., de Leval, M.R., Peek, G.J., Godden, P.J., Utley, M., Gallivan, S., Hirst, G. and Dale, T. (2006) Identification of Systems Failures in Successful Paediatric Cardiac Surgery. Ergonomics, 49, 567-588. https://doi.org/10.1080/00140130600568865

[30] Catchpole, K., Mishra, A., Handa, A. and McCulloch, P. (2008) Teamwork and Error in the Operating Room: Analysis of Skills and Roles. Annals of Surgery, 247, 699-706. https://doi.org/10.1097/SLA.0b013e3181642ec8

[31] Morris, J.A.J., Carrillo, Y., Jenkins, J.M., Smith, P.W., Bledsoe, S., Pichert, J. and White, A. (2003) Surgical Adverse Events, Risk Management, and Malpractice Outcome: Morbidity and Mortality Review Is Not Enough. Annals of Surgery, 237, 844-851.

[32] Takala, R.S., Pauniaho, S.L., Kotkansalo, A., Helmiö, P., Blomgren, K., Helminen, M., Kinnunen, M., Takala, A., Aaltonen, R., Katila, A.J., Peltomaa, K. and Ikonen, T.S. (2011) A Pilot Study of the Implementation of WHO Surgical Checklist in Finland: Improvements in Activities and Communication. Acta Anaesthesiologica Scandinavica, 5, 1206-1214. https://doi.org/10.1111/j.1399-6576.2011.02525.x

[33] Helmiö, P., Blomgren, K., Takala, A., Pauniaho, S.L., Takala, R.S. and Ikonen, T.S. (2011) Towards Better Patient Safety: WHO Surgical Safety Checklist in Otorhinolaryngology. Clinical Otolaryngology, 36, 242-247. https://doi.org/10.1111/j.1749-4486.2011.02315.x

[34] Böhmer, A.B., Wappler, F., Tinschmann, T., Kindermann, P., Rixen, D., Bellendir, M., Schwanke, U., Bouillon, B. and Gerbershagen, M.U. (2012) The Implementation of a Perioperative Checklist Increases Patients' Perioperative Safety and Staff Satisfaction. Acta Anaesthesiologica Scandinavica, 56, 332-338. https://doi.org/10.1111/j.1399-6576.2011.02590.x

[35] McConnell, D.J., Fargen, K.M. and Mocco, J. (2012) Surgical Checklists: A Detailed Review of Their Emergence, Development, and Relevance to Neurosurgical Practice. Surgical Neurology International, 3, 2. https://doi.org/10.4103/2152-7806.92163

[36] Byrnes, M.C., Schuerer, D.J., Schallom, M.E., Sona, C.S., Mazuski, J.E., Taylor, B.E., McKenzie, W., Thomas, J.M., Emerson, J.S., Nemeth, J.L., Bailey, R.A., Boyle, W.A., Buchman, T.G. and Coopersmith, C.M. (2009) Implementation of a Mandatory Checklist of Protocols and Objectives Improves Compliance with a Wide Range of Evidence-Based Intensive Care Unit Practices. Critical Care Medicine, 37, 27752781. https://doi.org/10.1097/CCM.0b013e3181a96379

[37] Windsor, J.A. and Petrov, M.S. (2010) Patient Safety in Medicine: Are Surgeons Ready for Checklists? ANZ Journal of Surgery, 80, 3-5. https://doi.org/10.1111/j.1445-2197.2009.05098.x

[38] Wilson, I. and Walker, I. (2009) The WHO Surgical Safety Checklist: The Evidence. Journal of Perioperative Practice, 19, 362-364.

[39] Hales, B.M. and Pronovost, P.J. (2006) The Checklist-A Tool for Error Management and Performance Improvement. Journal of Critical Care, 21, 231-235.

https://doi.org/10.1016/j.jcrc.2006.06.002

[40] Borchard, A., Schwappach, D.L., Barbir, A. and Bezzola, P. (2012) A Systematic Review of the Effectiveness, Compliance, and Critical Factors for Implementation of Safety Checklists in Surgery. Annals of Surgery, 256, 925-933. https://doi.org/10.1097/SLA.0b013e3182682f27

[41] Paige, J.T., Aaron, D.L., Yang, T., Howell, D.S., Hilton, C.W., Cohn, I.J. and Chauvin, S.W. (2008) Implementation of a Preoperative Briefing Protocol Improves Accuracy of Teamwork Assessment in the Operating Room. Annals of Surgery, 74, 
$817-823$

[42] Einav, Y., Gopher, D., Kara, I., Ben-Yosef, O., Lawn, M., Laufer, N., Liebergall, M. and Donchin, Y. (2010) Preoperative Briefing in the Operating Room: Shared Cognition, Teamwork, and Patient Safety. Chest, 137, 443-449.

https://doi.org/10.1378/chest.08-1732

[43] Sewell, M., Adebibe, M., Jayakumar, P., Jowett, C., Kong, K., Vemulapalli, K. and Levack, B. (2011) Use of the WHO Surgical Safety Checklist in Trauma and Orthopaedic Patients. International Orthopaedics, 35, 897-901.

https://doi.org/10.1007/s00264-010-1112-7

[44] Makary, M.A., Sexton, J.B., Freischlag, J.A., Holzmueller, C.G., Millman, E.A., Rowen, L. and Pronovost, P.J.(2006) Operating Room Teamwork among Physicians and Nurses: Teamwork in the Eye of the Beholder. Journal of the American College of Surgeons, 202, 746-752. https://doi.org/10.1016/j.jamcollsurg.2006.01.017

\section{Abbreviations}

WHO: World Health Organization

\section{Submit or recommend next manuscript to SCIRP and we will provide best} service for you:

Accepting pre-submission inquiries through Email, Facebook, LinkedIn, Twitter, etc. A wide selection of journals (inclusive of 9 subjects, more than 200 journals)

Providing 24-hour high-quality service

User-friendly online submission system

Fair and swift peer-review system

Efficient typesetting and proofreading procedure

Display of the result of downloads and visits, as well as the number of cited articles

Maximum dissemination of your research work

Submit your manuscript at: http://papersubmission.scirp.org/

Or contact ojsst@scirp.org 